\title{
Gender Patterns in Hypertext Reading
}

\author{
Aristidis Protopsaltis ${ }^{*}, 1$ and Vassiliki Bouki ${ }^{2}$ \\ ${ }^{1}$ The Serious Games Institute, Coventry University, Technology Park, Innovation Village, Cheetah Road, Coventry, CV1 \\ $2 T L, U K$ \\ ${ }^{2}$ Department of Computer Science, University of Westminster, Harrow Campus, Watford Road, Harrow, HA1 3TP, UK
}

\begin{abstract}
The effect of gender in learning has often been the focus of research because of its potential implications in academic achievement. However, the effect of gender in hypertext reading has not been thoroughly investigated. The Web in general and the hypertext in particular has modified the way people access and use information. This paper reports the findings of an empirical study into gender differences in hypertext reading. The study focuses on text-based electronic documents. The study is a mixed method design, with the use of the think-aloud protocols and a between subjects experiment. It examines some original variables not previously studied comprehensively, such as coherence of transactions, sum of selected hyperlinks, and hyperlink location. Forty two participants (30 males and 12 females) read a hierarchically structured hypertext and then, all answered the same set of questions. The data consisted of reading times, comprehension scores, reading strategies, coherent links, hyperlink location, sum of hyperlinks, and the sum of read nodes. The results show that gender did not significantly affect any of the measured variables.
\end{abstract}

Keywords: Hypertext, hyperlinks, comprehension, gender, reading, reading strategies, coherence, presentation location, cognitive processes, information processing.

Is there a difference in online information processing between males and females? The answer to this question has both theoretical and practical implications. The theoretical implications refer to the research in areas related to gender and online literacy, gender and information processing and, gender and educational media. The practical implications refer to the design of online environments. Understanding gender differences, if any, is an important consideration when designing online learning material. According to Weimin et al., [1] demographic factors, such as age, gender, and culture, have significant impact on software use and therefore should be considered in design.

Gender has been identified as a strong predictor of many attitudes and behaviours that have implications in Web information seeking [2]. However, before we proceed we need to define gender. There is confusion in the use of the term especially in computer and information sciences. Both terms gender and sex are often used indistinctively in literature and researchers refer to participants as male-female and men-women. However, "gender can be thought of as the behavioural, cultural, or psychological traits associated with one sex" [3 p. 785-786], while the term sex refers to biological characteristics. "In the study of human subjects the term gender should be used to refer to a person's selfrepresentation as male or female" [3, p. 786]. Consequently, the terms gender, and male-female should be used in the field of computer and information science, since they refer to behavioural attributes of participants. Therefore, the terms gender, and male-female are used in this work.

*Address correspondence to this author at the Serious Games Institute, Coventry University, Technology Park, Innovation Village, Cheetah Road, Coventry, CV1 2TL, UK; E-mail: aprotopsaltis@gmail.com
Early research in Internet use found differences in the rates of males and females online connectivity, with the former more connected $[4,5]$. More recent data however, indicated that this gender gap in basic connectivity has disappeared [6]. Another area that research has focused on was the difference between males and females in their use of the medium for interpersonal communication [7, 8]. This body of work has found that females spend more time corresponding with personal ties than their male counterparts. Little substantive work, though, has investigated Internet related skills by gender [9] and even less, hypertext related skills. Thus, contrary to some views that "there is little reason for concern about sex inequalities in Internet access and usage now" [6 p. 111], we might find important differences in how attitudes to the Web, the intensity and frequency of use, and user skill, differ by gender, all factors relevant to how much different groups may benefit from Internet usage [9].

The literature on gender differences in academic achievement is extensive. It is widely accepted that females score higher on average than males on test of verbal abilities $[10,11]$, while males score higher on average than females on tests of mathematical ability [12]. Recent results though show that gender differences in math tests in elementary and high school, in the USA, have been disappearing [13] and in some cases females surpass males [14]. However, stereotype threat has been identified as a possible influence on the differences between males and females in certain topics. For example, Cadinu et al. [15], and Dar-Nimrod \& Heine [16] investigating mathematical achievement found that women under the stereotype threat reported more negative thoughts related to the test and mathematics when compared to the no threat condition. It was also found that stereotype threat created a decrease in performance, which correlated to an 
increase in negative thoughts [15]. Thus, it seems that stereotypes influence the way males and females perform in academic context.

A number of studies though found no difference between genders in various learning conditions [12, 17-20]. Research has also looked at the relation between gender and cognitive styles [21, 22], where difference between genders were no significant. Contrary to those results though, differences between genders in their spatial ability exist [23]. Gender differences in visuospatial abilities have been thoroughly reported and confirmed by experimental evidence [24]. However, further research showed that gender effects in visuospatial tasks are not homogeneous: the characteristics of the task may induce the magnitude of differences between males and females. It has been shown that gender differences in visuospatial working memory (VSWM) are larger in tasks requiring active elaboration of the material [25] and gender effects, favouring men, are significantly more pronounced in active tasks [26]. Hypertext documents require a more active approach towards information compared to traditional documents, since locating information is necessary. Thus gender differences may play an important role on their effectiveness as an information vehicle.

In addition, significant differences in abilities for learning in general of males and females has been reported in the literature [27-29]. It is likely these differences to be observed in Web-based learning just as they would in traditional learning environments. Gender based social relationships, interaction styles and inequities that exist in traditional learning situations correspond fairly closely to those found in computer-supported environments. Barrett and Lally [30] for example, identified common differences in the behaviour of male and female students in technology based instructions. These differences included self-reported levels of confidence in the ability to work successfully with technology, use of support systems and patterns of interaction. They found that females talked less, contributed less frequently, did not receive positive feedback to their contributions and did not appeal to the same sources of support. Similarly, Gunn and McSporran [31] found gender differences in motivation, confidence level, flexibility and access. Males stated that they were very confident, that they would enjoy using the online materials whereas females stated they were apprehensive about using the materials and about their overall ability for the technical aspects of the course. Another area in which the differences emerged is support mechanism that students utilize when they need to clarify something related to course materials. Females mainly send e-mails to the lecturer while males contact their peers directly. Furthermore, other results seem to suggest that there are various gender differences which were identified within learning from computer-based technologies [32]. There are a number of research studies that found gender differences in the learning outcomes. However, these studies showed mixed results. Some found that females are more successful in web-based learning [33], while others found that males performed better [34-36], and some others reported that there was no significant difference between genders in their learning ability [12, 17-20]. However, little is known about how users are able to make use of and learn from new technologies [37].
It is evident from the data discussed here that there are inconclusive results on the effect of gender on the use of Web published material. It is also apparent that there is a need for further research in the area of online documents and information processing. Questions still exist pertaining to whether or not males and females significantly differ in terms of their learning behaviour in general, and in terms of their reading and information processing behaviour in particular, in electronic environments. Studying such questions is important because, if gender disparities are found, they may lead teachers and researchers to seek ways to minimize them, thus affording both genders maximum opportunities to achieve high levels of learning, academic achievement, and technology use. Research so far has focused on the relation between gender and academic performance measuring primarily variables such as comprehension and amount of time spent on a given task. Electronic texts however, that incorporate hyperlinks and hypermedia, require skills and activities beyond those required for comprehension of conventional, linear textbooks [38]. These activities have not been vigorously investigated by researchers and especially in conjunction with gender, such skills are hyperlink location, amount of hyperlinks selected, coherence of the link selection etc. The current study seeks to shed more light in this area by investigating the effects that gender might have in reading and learning in hypertexts, examining a range of variables not previously comprehensively studied.

\section{METHOD}

\section{Subjects}

Forty two (42) undergraduate students, from the Harrow School of Computer Science (HSCS) and the Media Arts and Design (MAD) department of the University of Westminster participated. Twelve (12) participants were self-identified as female, while thirty (30) as male. All participants were volunteers. They were screened to ensure that they had not taken any courses in economics, and had no reading disabilities. All participants were native English speakers, apart from one, and all they were familiar with Web documents. None of the participants had participated in a think-aloud study before.

\section{Material: Reading and Comprehension}

A 5,075-word paper in economics converted to a hypertext [see: 39 for more details]. The subject matter was chosen to avoid any influence of background knowledge in comprehension. The focus of the study was on reading, thus the hypertext consisted of text only. The structure of the hypertext was based on the semantic structure provided by the author and it was converted to a hierarchical tree (see Fig. 1). A total of 23 nodes were created and 136 hyperlinks were available. There was a welcome page before the main document. A menu for navigational purposes was available at the left hand side of the hypertext. Users had a choice of global and local navigational links. Participants were given two types of comprehension tasks, multiple choice questions and short essay questions. There were twelve multiplechoice items, two open-ended questions and one essay question. Students were not penalized for errors of grammar, spelling, or punctuation. The highest possible comprehension score was twenty (20). 


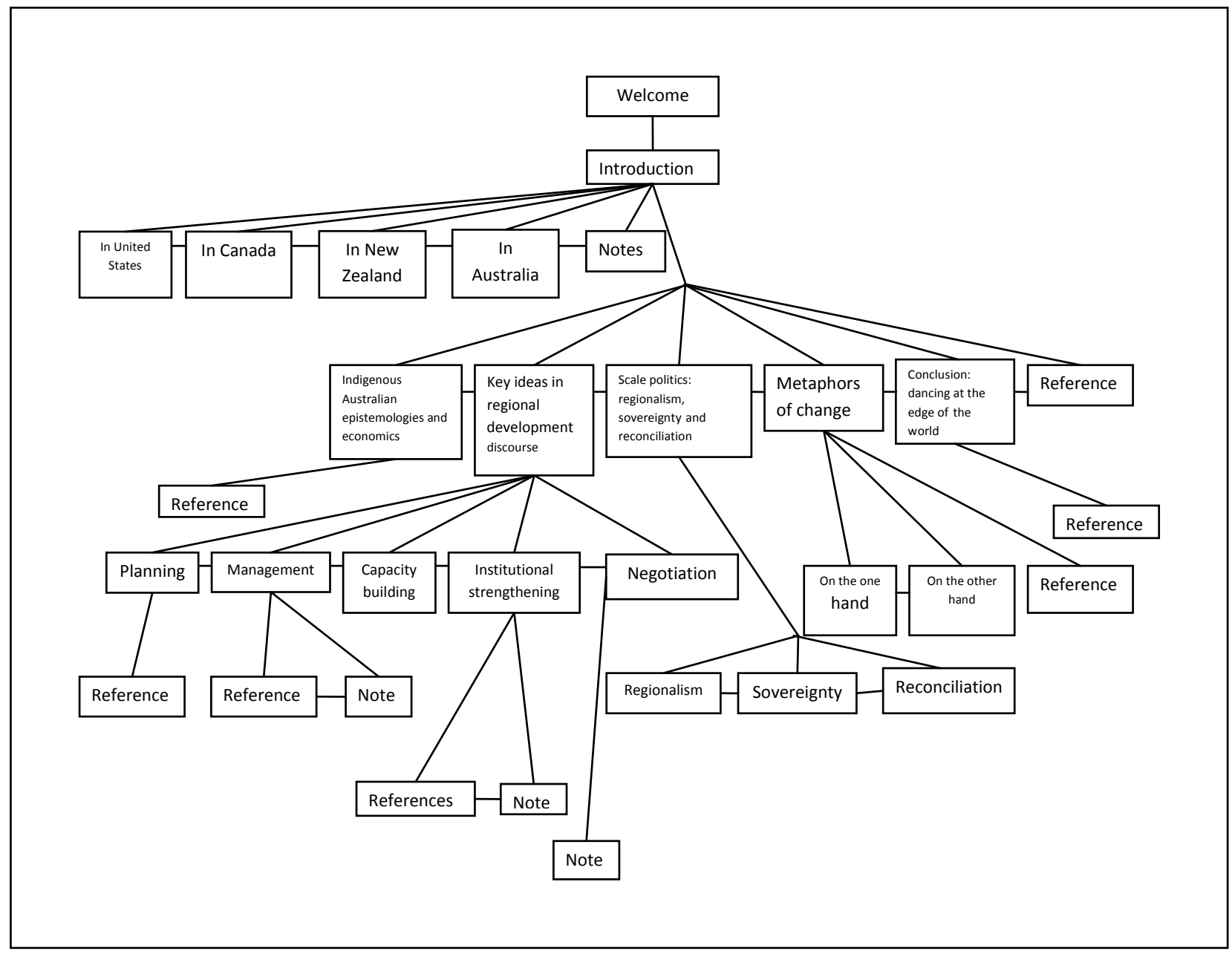

Fig. (1). Structure of the hypertext.

\section{Apparatus}

Personal computers were used to display the hypertext. A Pentium IV $1.8 \mathrm{GHz}$ Hi-Grade computer with 512MB memory was used. The monitor was CTX 17 inches Cathode Ray Tube (CRT) colour monitor. The monitors' resolution was $1024 \times 768$ pixels. A Java Script cookie was used to record the times and the readers' transitions in the hypertext document throughout the session. A SONY M-560V micro cassette recorder was used to record the think-aloud protocols.

\section{Design}

The study was a mixed methods experiment, using a between subjects design ( 3 by 1 ), manipulating the reading goals, and the think-aloud method. The reading goals were manipulated by providing different instructions about what the participants should read in the text. Simply instructing participants to read a text for normal comprehension does not even assure the comprehension has taken place. Therefore researchers attempt to assess participants' comprehension of a given text by asking them to summarise or to recall, or to answer questions about it, or even all of it together [40]. A previous analysis [39, 41, 42] on the data failed to reveal significant differences between the groups with different reading goals. Consequently the data were collapsed across gender, and as a result the only independent variable considered here is gender. Taking into account gender differences in visuospatial working memory and in learning, the experimental null hypothesis $\left(\mathrm{H}_{0}\right)$ predicts that gender will not affect participants reading times, comprehension scores, number of visited nodes, sum of hyperlinks, amount of coherent hyperlink transition, and the sequence of the hyperlink selection, while the alternate hypothesis $\left(\mathrm{H}_{1}\right)$ predicts that gender will affect all the variables.

\section{Procedure}

Participants were settled comfortably in a quiet room and a glass of water was provided. They were randomly assigned to one of the three conditions. They were briefly told the aim of the study and they read the text until they felt satisfied that they were able to answer questions on the subject matter. Warm up exercises were given for practicing the think-aloud method until they felt confident with it. After the reading task, participants received the booklet with the recognition material. All participants answered the same set of questions without consulting the learning material. The experiment 
was conducted in individual sessions. Each session took about one hour to be completed.

\section{RESULTS}

\section{Reading Times}

The reading times and amount of the selected links received from the time-stamped hypertext records, while the comprehension scores were calculated from the comprehension booklet. To examine those results a one-way analysis of variance (ANOVA) was conducted. The total time to read the hypertext was recorded by a Java Script cookie. The mean time to read the hypertext was 35.2 minutes with a standard deviation of 12.3 (see Table 1).

Table 1. Means and Standard Deviations of Reading Times

\begin{tabular}{|c|c|c|c|c|c|}
\hline & N & Minimum & Maximum & Mean & Std. Deviation \\
\hline \hline Reading Times & 42 & 8.00 & 59.00 & 35.1905 & 12.28570 \\
\hline Gender & 42 & 1.00 & 2.00 & 1.2857 & .45723 \\
\hline Valid N & 42 & & & & \\
\hline
\end{tabular}

There was no significant difference between the reading times based on the different gender $(\mathrm{F}(1,40)=.004$, $\mathrm{p}=.950$ ). A box plot analysis (see Fig. 2) showed that the majority of the scores for both males and females were close to the mean. However, the data showed that males had more extreme scores compared to females.

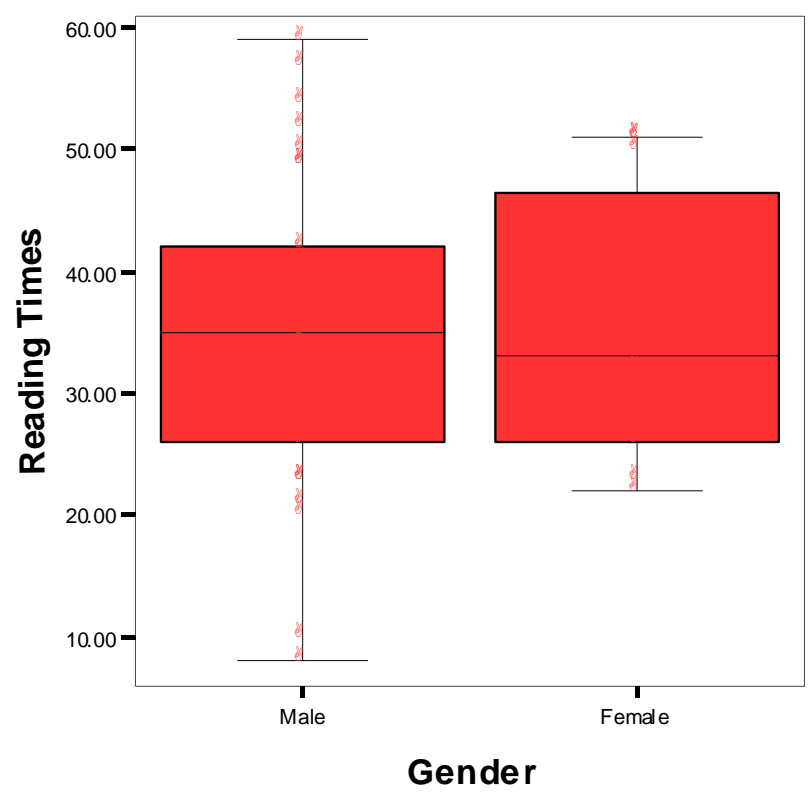

Fig. (2). Distribution of reading times.

\section{Comprehension Scores}

One type of measuring comprehension was obtained. The multiple choice and the short answer questions were graded and one score for each subject was calculated. The maximum achievable score was 20. A box plot analysis (see Fig. 3) showed that males had more extreme scores outside both the upper and lower quartile compared to females. However, there was no significant difference in comprehension based on the different gender $(\mathrm{F}(1,40)=2.130, \mathrm{p}=.152)$.

\section{Visited/Read Nodes}

The amount of the selected links received from the timestamped hypertext records. The amount of the selected links by the participants signifies another approach to gain an insight about hypertext reading. That approach shows, if participants have taken advantage of the hypertext features that permit them to locate specific subsets of information quickly. The mean percentage of the nodes per gender is 17.1 nodes for males and 17.3 for females (see Fig. 4). A node was considered visited, if a subject had selected the node at least once. Thus all the visited nodes are considered as read. However, if a node was visited more than once it was not counted as a different visited node. The number of nodes visited by the participants was revealed by a Java Script cookie. The total number of hypertext nodes read by participants was calculated. The maximum amount of visited nodes that a subject could read was 23. Participants visited/read 731 nodes in total. The range of visited nodes varies between 2 and 22 as Fig. (5) shows. There was no significant difference between the hypertext nodes that the participants read based on the different gender $(\mathrm{F}(1,40)=$ $.026, \mathrm{p}=.873)$.

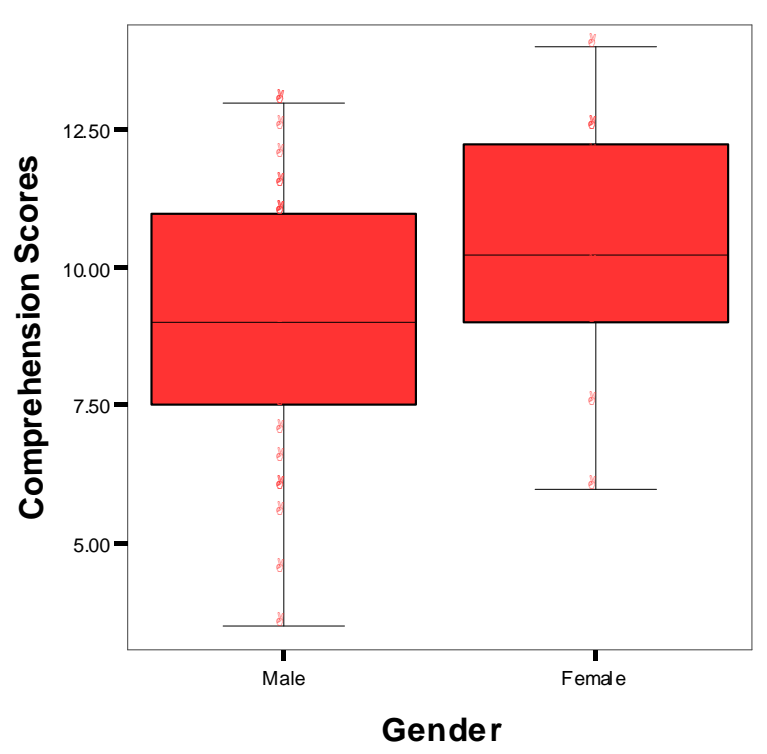

Fig. (3). Distribution of comprehension scores.

\section{Strategies}

The strategies that hypertext readers used during reading were made known by the think-aloud protocols. The analysis of the participants' transcripts revealed four strategies: a serial strategy, a serial overview strategy, a mixed strategy, and a mixed overview strategy $[39,42]$. There was no significant difference between the hypertext nodes that the participants read based on the different gender $(\mathrm{F}(1,40)=$ $.027, \mathrm{p}=.870$ ). A post hoc test was conducted (Tukey HSD) to examine if there is any significant difference between the groups of the different strategies based on their gender. There was no significant difference between groups $(\mathrm{p}=$ 
.093 ). However, $10 \%$ more females ( $50 \%$ overall) used the most complex from the strategies, the mixed overview, compared to their males' counterparts (40\%). Furthermore, females did not use the serial overview strategy whatsoever, while they used the serial strategy much more $(33.33 \%)$ compared to males (13.33\%) (see Fig. 6).

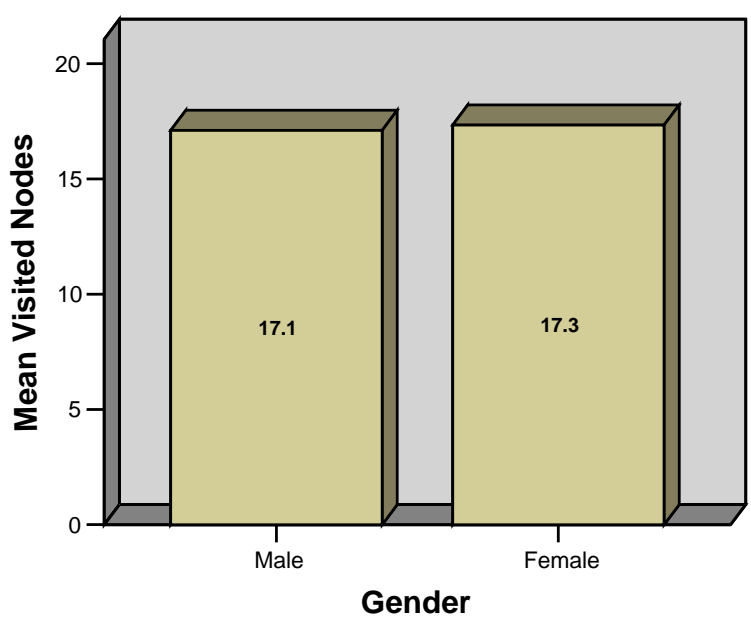

Fig. (4). Means of visited nodes per condition.

\section{Factors Influencing Navigation Strategies}

The rules participants used to get to the different nodes of the hypertext need to be considered in order to improve reader behaviour and development of electronic documents.
In a previous analysis of the think-aloud protocols [39, 41, 42] three such rules were revealed: coherence, personal interest, and link position. However, there is not enough data to investigate the personal interest since only a few participants verbalised their interest. Thus the present study investigated the effect that gender might have on only two factors, the coherence and the location of the links.

\section{Coherence}

Coherence is a fundamental characteristic of comprehension in traditional documents. A coherent transition in the hypertext was considered a transition from one node to another in which both nodes were still within the same context. This included such cases as jumping to a parent, or child or sibling node of the current node. In addition, cases such as following the presentational order of the nodes, which is closely related to the hierarchical structure, were considered coherent transitions [39, 41, 42]. The examination of coherence focused on the first time that readers accessed/read a node, and thus each transition was counted only once. The participants' transitions were extracted from the JavaScript cookie records kept in the server. Participants selected 730 hypertext links in total. They made $93.70 \%$ of their transitions in a coherent way regardless of their gender. Both genders made identical coherent transactions. Male participants made 16.1 coherent hyperlink transitions while female participants made 16.75 (see Fig. 7) coherent hyperlink transitions. There was no significant difference between the gender and the coherence of the hyperlink selection $(\mathrm{F}(1,40)=3.621 \mathrm{p}=.692)$.

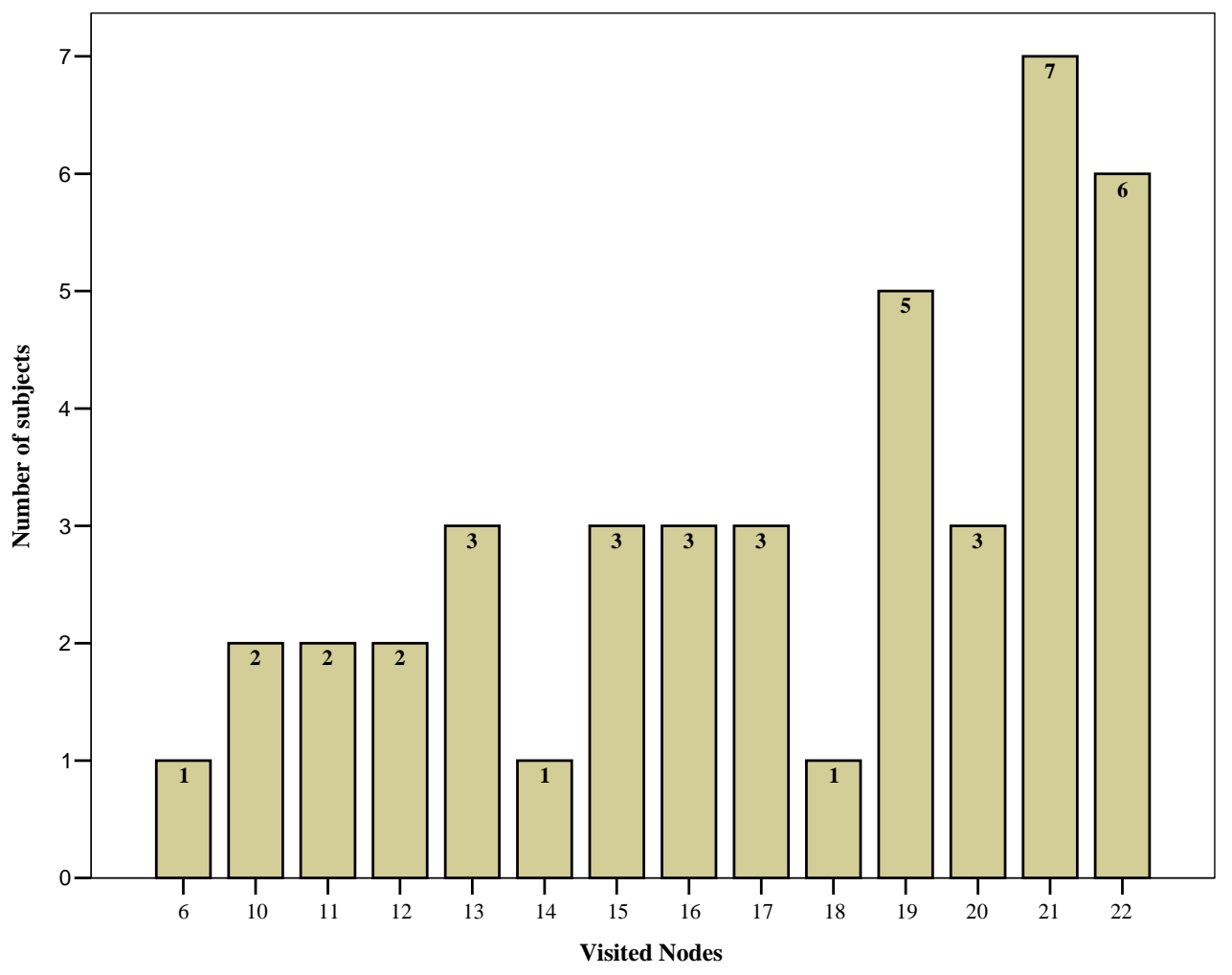

Fig. (5). Range of visited nodes. 


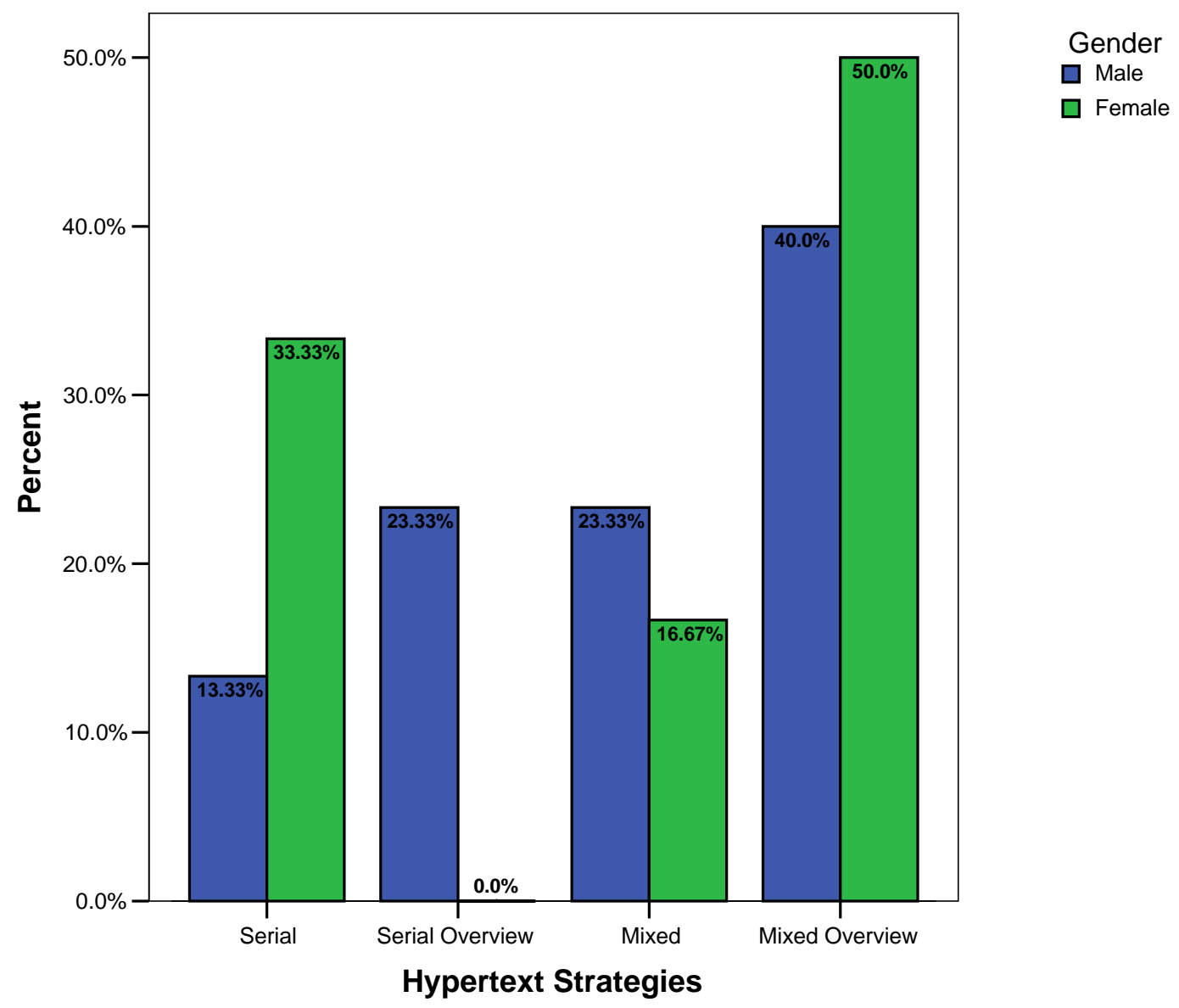

Fig. (6). Means of coherent transitions per condition.

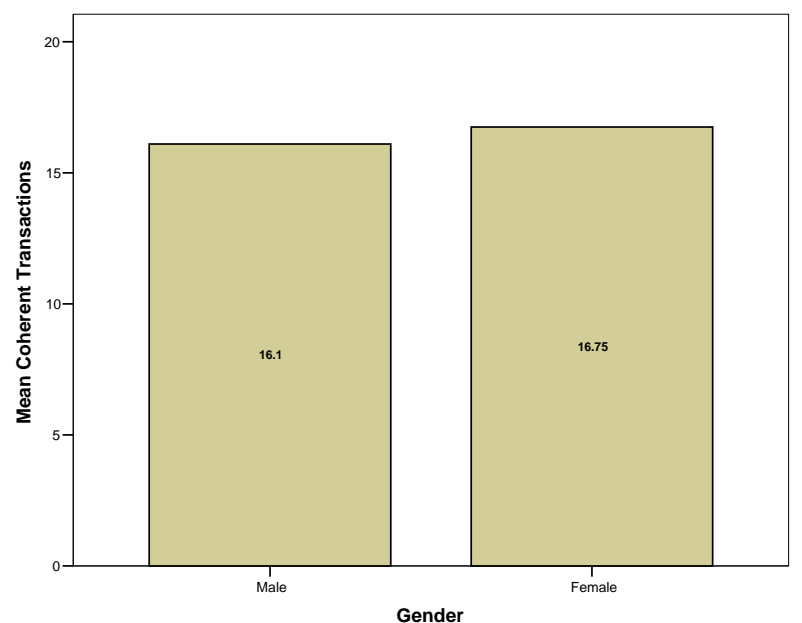

Fig. (7). Means of coherent transitions.

\section{Presentation Location}

Previous analysis [39, 41, 42] revealed that the location of the links influences its selection. The location was defined by the linguistic sequence, starting from left to right and top to bottom, following the regular reading pattern. Users tended to select the first available link while reading. Every

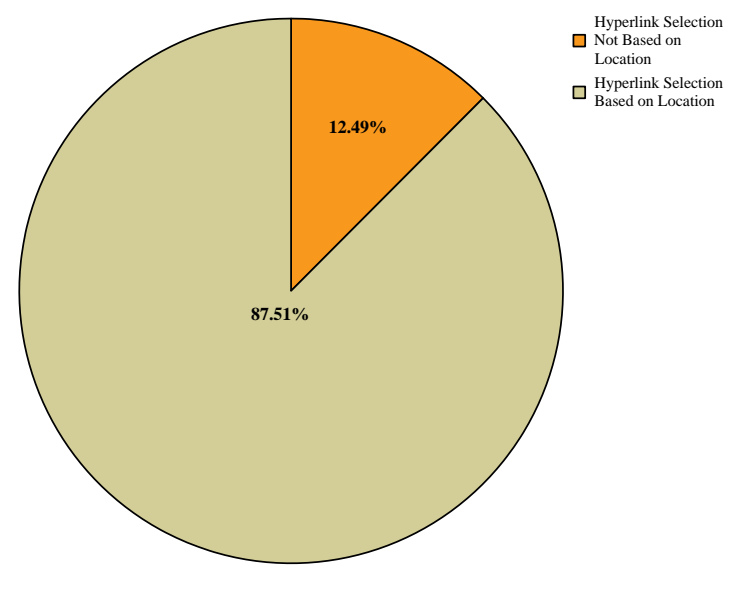

Fig. (8). Location-based transitions.

selection of hypertext links was considered and counted, including those hyperlinks that were selected more than once. The 42 participants produced a sum of 865 hyperlink selections. From those hyperlink selections $87.51 \%$ were made based on their location (see Fig. 8). Hence, participants selected those links as soon as they encounter them following their reading pattern. The $12.49 \%$ of the hypertext 
link selections were made in a different way not based on their positioning in the hypertext. Male participants selected 17.2 hyperlinks based on their location while female participants made 20.08 location-based hyperlink transitions on average (see Fig. 9). Females made more location-based hyperlink selection compared to males but the difference was no significant $(\mathrm{F}(1,40)=1.347 \mathrm{p}=.253)$.

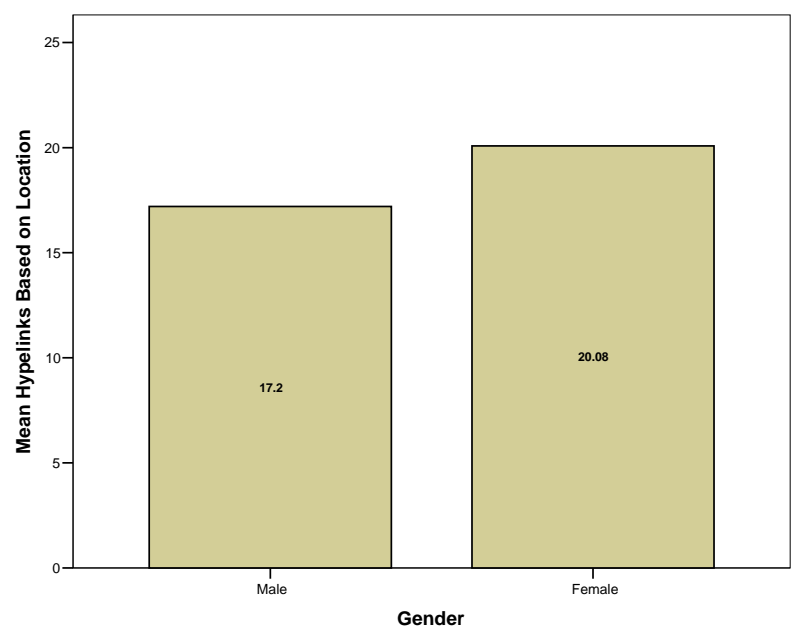

Fig. (9). Selection of hyperlinks based on location.

\section{DISCUSSION}

The current study investigated the effect of gender on hypertext information processing, studying a range of variables, some of which have not been vigorously examined before. The results suggested that there is no significant difference in any of the measured variables; forcing us to accept the null hypothesis, that gender does not influence any of the measured variables. The results coincide with those obtained by the authors in an other study [20], using a different experimental method, with larger sample of participants. The fact that, two different methods produced similar results provides extra validity in the findings. Similarly, other studies $[12,17,18]$ did not reveal any significant difference in performance between genders. However, the current findings are in disagreement with other studies, some favouring males [36, 43-45] and other favouring females [46, 47].

The issue of gender differences in relation to computerbased learning and use of technology has generally centred around findings that suggest that males tend to demonstrate more positive attitudes and learning outcomes than females [36, 43-45]. Such differences have sometimes been attributed to a propensity for males to have greater experience with using computers $[48,49]$. However, in the current study all participants had similar computer and Web experience since the majority of them were self-identified as experienced users apart from 2 females and 4 males. This balance on experience might have contributed to the lack of any significant difference in hypertext use, since experience and exposure on the Web is one of the factors that influence gender performance in online material, favouring males.

The current findings revealed no significant difference in reading times between genders, showing that males and females spent roughly the same time reading the hypertext.
These results are in conflict with other findings that showed that females were more thorough in reading Web pages and took more time in completing their tasks [50]. In the same vein, Large, et al. [47] in their study found that females spent more time reading online documents than their male counterparts. However, such a difference might be due to the sample differences since their study was based on school pupils', while the current one was based on university students where their exposure on the Web is greater, and thus their strategies might be more effective. Another reason for such differences can be the fact that, in the current study participants had to search for and read information in a restricted hypertext environment, whereas in the other studies [47, 50] participants had more freedom and flexibility in using more than one Web document. Contrary to these studies though, the current findings revealed that males had more extreme scores compared to females, although the difference was not significant. In a follow up study though [20] no such effect was observed. This might be because of the different experimental methods used in the two studies.

Similar results were found regarding comprehension. There was no significant difference between males and females in comprehension scores. These results are in conflict with the commonly believed notion that females score higher on average than males on test of verbal abilities [11]. However, the results for comprehension in the field are mixed, since some researchers have found that males performed better than females [34-36]. Roy and Chi [51] found that young males performed significantly higher than young females on both, target-specific and target-related information measures. Other researchers though have found that females were more successful in Web-based learning [33], and others have found no difference between genders $[12,17-20]$, similar to the current study.

Regarding the strategies, participants used similar strategies since there was no significant difference between males and females on their strategies. These results are in line with results reported by Alexander Poole [52]. His study nonetheless focused on the use of strategies in second language learning (L2). However, one notable result in the present study is the fact that $10 \%$ more females $(50 \%)$ used the most complex from the strategies, the mixed overview, compared to their male counterparts $(40 \%)$. Additionally, females did not use the serial overview strategy whatsoever, while they used the serial strategy much more $(33.33 \%)$ compared to males (13.33\%). Roy et al. [37] found differences in the use of search strategies with females demonstrating proportionally more vertical movement. They noted that such results suggest that young females were much more linear and thorough navigators than young males. Their results are partially consistent with the ones found here since females seem to use linear strategies more than males, however, not significantly more. Nevertheless, more research is needed in the area of hypertext strategies, with a larger sample of participants and especially a larger number of female participants in order to produce more defined results.

Another way to gain an insight about reading behaviour in hypertext environments is to investigate the amount of visited nodes. The number of visited/read nodes are very 
closely related to reading comprehension because it is affecting the formation of the text-base [53]. Text-base is defined as the restricted meaning of the text, and is narrowed down to the level of individual sentences and paragraphs [54, 55]. The present study provides some unique results regarding different aspects of online information processing. The findings showed no significant difference between the gender and the amount of visited nodes by readers. However, the results are not surprising since both genders spent roughly the same time to read the hypertext and had similar comprehension scores, implying that they visited similar number of nodes. These results coincide with the findings obtained in another study [56], using a different method, where it was found that there was no difference between gender and the amount of visited nodes. Similarly, Thewall et al. [57] found little evidence of gender differences on link selection. Large, et al. [47] on the other hand, found males to be generally more active in the way they searched the Web, clicking on more links per minute, and following up on more hits. Similarly, Roy and Chi [51] found that the overall pattern of search behaviour was different for males and females. Similarly, Vecchi and Girelli [26] explored gender differences in visuospatial tasks that either required memorizing (passive task) or manipulating and transforming (active task) visuospatial information. Their results showed that gender effects, favouring men, were significantly more pronounced in active tasks. However, no such claims can be made from the current study since, both genders behaved in a similar way in an active task. One reason for such difference might be the fact that the primary focus of these studies [26, 47] was on searching the web, while in the present study reading was the focal point.

The findings regarding the coherence of information and location of the links revealed that gender did not affect the amount of coherent transactions and the selection of hyperlinks based on the location. Both male and female participants made identical coherent transitions with 16.1 and 16.75 respectively. Although the hypertext permitted non-coherent transitions, by offering easy access to the other hyperlinks, both genders chose to make their transitions in a coherent manner. These results emphasise the importance of coherence in reading and understanding regardless the reading/learning medium. Therefore, textual cohesion is equally important in hypertexts [58] as in paper-based documents. These results coincide with those obtained in another study [20] run by the authors.

The location of the links seemed to be a decisive factor in selecting hyperlinks, since it influenced almost ninety per cent $(87.51 \%)$ of the hyperlink selection for both genders. The results suggested that the position of the links in the hypertext, directly affects their selection. The current data refer to the first selection only based on the location, and it is significant because of its possible implications on the hypertext structure. Participants tended to select the first link they came across from left to right and also from top to bottom. For example, when readers had to read information lying underneath some links grouped together in a bullet point format, they primarily chose to start with the top link, and continued with the one straight below. Yet, further research is needed because these results might have been influenced by the experimental design or the hypertext structure. In a follow up study [20], the influence of the location of the links was still very influential affecting more than half $(55.38 \%)$ of the hyperlink selection for both genders. However, the percentage was considerably lower compared to the current study. This difference might be due to the fact that in the second experiment the results included revisited links, which might differ in the way they were accessed. The purpose of revisiting was mainly for refreshing the information previously read, and participants tended to access them in a more random way. Readers tended to review the information at the end of their reading much more by revisiting the hypertext nodes and skimming through the information. Thus the current results suggest that there is a direct relationship between the position of a hyperlink and its probability of been selected. Therefore we could argue that the location of the links in hypertexts greatly affects its selection sequence and it needs to be considered by the designers. Readers were more comfortable selecting the links following their linguistic sequence, rather than breaking away and selecting the hyperlinks in a different way. Such results coincide with well documented learning practices where information needs to be wellstructured for novice learners [59]. Likewise, Ainley et al. [60] found that approximately $50 \%$ of the participants in their study just read the text following the order in which they were presented on the screen. This indicates that both male and female were more comfortable selecting the links following its linguistic sequence, rather than breaking away and selecting the links in a different way. Participants tended to select the first link they came across from left to right and also from top to bottom. The present results are consistent with results obtained by McDonald and Spencer [61]. They found no significant differences between males and females in navigational behaviour. Furthermore, Klöckner, et al. [62] found that the sequence of links is a decisive factor in hyperlink selection when users examined search results before opening a document. Selecting the hypertext links based on their location seems to offer great support to both genders. Charney [63] argues that a predefined sequence plays an important role in text comprehension processes because readers tend to consider early information as important, and they are also sensitive to textual cohesion. Subsequently, predefined sequence seems to be very important for readers in electronic environments as well, and influences their navigational choices notably. Another reason for the use of linear strategies by the participants can be the lack of hypertext reading strategies. Reading and learning in academic context is based primarily on traditional text books and thus readers when they have to use new media they borrow strategies that they feel comfortable with and not develop new ones, which might be more effective when using new hypermedia.

\section{CONCLUSION}

The current study examined the effects of gender on hypertext information processing and shaded some light on variables not thoroughly investigated before. Our data suggested that male and female web users do not significantly differ in their reading comprehension, reading times, coherent hyperlink selection, hyperlink location; sum of hyperlinks selected and amount of visited nodes in electronic environments. The study offers an original perspective regarding some reading aspects exclusive to 
electronic documents such as, hyperlink selection, hyperlink location, sum of hyperlinks selected and amount of visited nodes in electronic environments. It has offered a more comprehensive view in the relation between gender and information processing. However, the findings add into a body of knowledge with mixed results. As a result, they yell the need for more research. Further empirical research is essential in order to draw unambiguous conclusions on the effects that gender might have in online information processing. This is important, if we want to provide the best possible support to users of electronic information platforms and help them to make optimum use of the new mediums.

\section{REFERENCES}

[1] Weimin H, Manpreet K, Anita K, et al. "Girls don't waste time": pre-adolescent attitudes toward ICT. In: CHI '06 Conference on human factors in computing systems; 2006 April 22-27; Montreal, Quebec, Canada: ACM Press 2006; pp. 875-80.

[2] Hupfer EM, Detlor B. Gender and web information seeking: a selfconcepted orientation model. J Am Soc Inf Sci Technol 2006; 57(8): 1105-15.

[3] Torgrimson BN, Minson CT. Sex and gender: what is the difference? J Appl Phys 2005; 99: 785-7.

[4] Bimber B. The Gender gap on the internet. Soc Sci Q 2000; 81: 868-76.

[5] Denis A, Ollivier M. How wired are Canadian women? the intersection of gender, class and language with the use of new information technologies 2002 .

[6] Ono H, Zavodny M. Gender and the internet. Soc Sci Q 2003; 84: $111-21$.

[7] Boneva B, Kraut R, Frohlich D. Using e-mail for personal relationships: the difference gender makes. Am Behav Sci 2001; 45: 530-49.

[8] Kennedy T, Wellman B, Klement K. Gendering the digital divine. IT Society $2003 ; 1: 149-72$.

[9] Hargittai E, Shafer S. Differences in actual and perceived online skills: the role of gender. Soc Sci Q 2006; 87(2): 432-48.

[10] Dee T. The why chromosome: how a teacher's gender affects boys and girls. Educ Next 2006; 4: 69-75.

[11] Hyde JS, Linn MC. Gender differences in verbal ability: a metaanalysis. Psychol Bull 1988; 104: 53-69.

[12] Hyde JS, Fennema E, Lamon JS. Gender differences in mathematics performance: a meta-analysis. Psychol Bull 1990; 107: 139-55.

[13] Glazer S. Gender and learning. CQ Res 2005; 15(19): 445-68.

[14] Halpern DF. A cognitive process taxonomy for sex differences in cognitive abilities. Curr Dir Psychol Sci 2005; 13(4): 134-9.

[15] Cadinu M, Maass A, Rosabianca A, Kiesner J. Why do women underperform under stereotype threat? Psychol Sci 2005; 16(7): 572-8.

[16] Dar-Nimrod I, Heine JS. Exposure to scientific theories affects women's math performance. Science 2006; 314(5798): 435

[17] Astleitner H, Steinberg R. Are there gender differences in webbased learning? an integrated model and related effect sizes. AACE J 2005; 13(1): 47-63.

[18] Anderson RE. Females surpass male in computer problem solving: findings from the minnesota computer literacy assessment. J Educ Comput Res 1987; 3: 39-51.

[19] Protopsaltis A, Bouki V. Does gender matter in hypertext reading? In: ED-MEDIA 2008 world conference on educational multimedia, hypermedia \& telecommunications; 2008 June 30 - July 4; Vienna, Austria: Chesapeake, VA: AACE 2008; pp. 1120-9.

[20] Protopsaltis A, Bouki V. Reading and gender in electronic age. In: ACM SIGDOC 26th international conference on design of communication; 2008; Lisbon, Portugal: ACM Press 2008; pp. 131-8.

[21] Riding R, Grimley M. Cognitive style, gender and learning from multi-media materials in 11-year-old children. Br J Educ Technol 1999; 30(1): 43-56.

[22] Riding RJ. Cognitive style: a review. In: Riding RJ, Rayner SG, Eds. International perspectives on individual differences. Stanford CT: Ablex; 2000; pp. 315-44.
[23] Linn MC, Paterson AC. A meta-analysis of gender differences in spatial ability: implications for mathematics and science achievement. In: Hyde JS, Linn MC, Eds. The psychology of gender: advances through meta-analysis. Baltimore: John Hopkins University Press 1986.

[24] Andrea B, Longoni MA, Vecchi T. Gender effects in spatial orientation: cognitive profiles and mental strategies. Appl Cogn Psychol 2004; 18: 519-32.

[25] Cattaneo Z, Postma A, Vecchi T. Gender differences in memory for object and word locations. Q J Exp Psychol 2006; 59: 904-19.

[26] Vecchi T, Girelli L. Gender differences in visuo-spatial processing: the importance of distinguishing between passive storage and active manipulation. Acta Psychol 1998; 99: 1-16.

[27] Halpern DF. Sex differences and cognitive abilities. Mahwah: NJ: Lawrence Erlbaum 2000.

[28] Weiman H. Gender differences in cognitive functioning. 2001 [Retrieved 2001 20/12]; Available from: http://faculty.kendall.edu/ hweiman/GenderDifferences.html

[29] Whitley BEJ. Gender differences in computer related attitudes and behavior: a meta-analysis. Comput Hum Behav 1997; 13: 1-22.

[30] Barrett E, Lally V. Gender differences in an online learning environment. J Comput Assist Learn 1999; 15: 48-60.

[31] Gunn C, McSporran M. Dominant or different? gender issues in computer supported learning. J Asynchr Learn Netw 2003; 7(1): 14-39.

[32] Lal V. Gender and the impact of computer-based technologies 2002 [Retrieved 2002 27/11]; Available from: http://www.effects project.uts.edu.au/project_papers/gender_cbl.pdf

[33] Mehlenbacher B, Miller CR, Covington D, Larsen JS. Active and interactive learning online: a comparison of web-based and conventional writing classes. IEEE Trans Prof Commun 2000; 43(2): 166-84

[34] McSporran M, Young S. Does gender matter in online learning? Assoc Learn Technol J 2001; 9(2): 3-15.

[35] McSporran M, Young S, Dewstow R. Who wants to learn online? Identifying our flexible learners. In: Proceedings of the world conference on educational multimedia, hypermedia \& telecommunications conference; 1999; Seattle, Washington, USA: AACE 1999; p. 1553.

[36] Alkhalifa E. The effects of using a bulletin board on gender differences in program coding. AACE J 2008; 16(2): 95-114.

[37] Roy M, Taylor R, Chi TH, Michelene. searching for information on-line and off-line: gender differences among middle school students. J Educ Comput Res 2003; 9(2): 229-52.

[38] Rand RSG. Reading for understanding: toward an R\&D program in reading comprehension. Santa Monica: RAND Reading Study Group 2002.

[39] Protopsaltis A. Reading strategies in hypertexts and factors influencing hyperlink selection. J Educ Multim Hyperm 2008; 172(2): 190-213.

[40] Ericsson KA. Concurrent verbal reports on text comprehension: a review. Text 1988; 8(4): 295-325.

[41] Protopsaltis A, Bouki V. The effects of reading goals in hypertexts reading. ACM SIGDOC 24th international conference on design of communication; 2006; South Carolina, USA 2006; pp. 29-34.

[42] Protopsaltis A, Bouki V. Reading strategies in hypertexts and factors influencing link selection. ED-MEDIA 2006 world conference on educational multimedia, hypermedia \& telecommunications; 2006; Orlando, Florida: AACE 2006; pp. 4507.

[43] Djordje K. Gender differences in computer attitude among ninthgrade students. J Educ Comput Res 2000; 22(2): 145-54.

[44] Todman J, Dick G. Primary children and teachers' attitudes to computers. Comput Educ 2000; 20(2): 199-203.

[45] Schumacher P, Morahan-Martin J. Gender, internet and computer attitudes and experiences. Comput Hum Behav 2001; 17(1): 95110.

[46] Light P, Littleton K, Bale S, Joiner R, Messer D. Gender and social comparison effects in computer-based problem solving. Learn Instr 2000; 10: 483-96.

[47] Large A, Beheshti J, Rahman T. Gender differences in collaborative Web searching behavior: an elementary school study. Inf Proc Manage 2002; 38(3): 427-43.

[48] Mitra A, Steffensmeier T, Lenzmeier S, Avon R, Qu N, Hazen M. Gender and computer use in an academic institution: report from a longitudinal study. J Educ Comput Res 2000; 23(1): 67-84. 
[49] Nachmais R, Mioduser D, Shemla A. Information and communication technologies usage by students in an Israeli high school: Equity, gender, and inside/outside school learning issues. Educ Inf Technol 2001; 6(1): 43-53.

[50] Leong SC, Hawamdeh S. Gender and learning attitudes in using web-based science lessons. Inf Res 1999; 5.

[51] Roy M, Chi MTH. Gender differences in patterns of searching the web. J Educ Comput Res 2003; 29(3): 335-48.

[52] Poole A. Gender differences in reading strategy use among ESL college students. J Coll Read Learn 2005; 36(1): 7-20.

[53] Salmerón L, Kintsch W, Cañas J. Reading strategies and prior knowledge in learning from hypertext. Mem Cognit 2006; 34(5): 1157-71.

[54] Kintsch W. The use of knowledge in discourse processing: a construction-integration model. Psychol Rev 1988; 95: 363-94.

[55] van Dijk TA, Kintsch W. Strategies of discourse comprehension. London: Academic Press 1983.

[56] Protopsaltis A, Bouki V. Gender patterns in hypertext reading. In: Workshop on cognition and the web. Granada, Spain, 24-26 April, 2008; pp. 213-7.
[57] Thelwall M, Barjak F, Kretschmer H. Web links and gender in science: an exploratory analysis. Scientometrics 2006; 67(3): 37383.

[58] Storrer A. Coherence in text and hypertext. Doc Des 2002; 3(2): $156-68$

[59] Dillon A. Myths, Misconceptions, and an alternative perspective on information usage and the electronic medium. In: Rouet J, Levonen JJDA, Spiro R, Eds. hypertext and cognition: Lawrence Erlbaum Associates 1996; pp. 25-42.

[60] Ainley M, Hidi S, Berndorff D. Interest, learning, and the psychological processes that mediate their relationship. J Educ Psychol 2002; 86: 303-11.

[61] McDonald S, Spencer L. Gender differences in web navigation. In: Balka E, Smith KR, Eds. Computerization: charting a course to the future. Boston: Kluwer Academic Publishers 2000; pp. 174-81.

[62] Klöckner K, Wirschum N, Jameson A. Depth- and breadth-first processing of search result lists. CHI '04 extended abstracts on Human factors in computing systems. Vienna, Austria: ACM 2000

[63] Charney D. Comprehending non-linear text: the role of discourse cues and reading strategies. In: Weiss S, Schwartz S, Eds. Proceedings of hypertext '87; 1987: Chapel Hill: ACM 1987; pp. 109-20.

(C) Protopsaltis and Bouki; Licensee Bentham Open.

This is an open access article licensed under the terms of the Creative Commons Attribution Non-Commercial License (http: //creativecommons.org/licenses/bync/3.0/) which permits unrestricted, non-commercial use, distribution and reproduction in any medium, provided the work is properly cited. 